\title{
Aspects of a Thermodynamic Approach to Phase Diagram Calculations
}

\author{
I. Ansara, Saint Martin-d'Hères \\ Laboratoire de Thermodynamique et Physico-Chimie Métallurgiques
}

In 1957, Meijering ${ }^{1)}$ published the first calculated isothermal section of a ternary phase diagram, namely that for the coppernickel-chromium system, using a thermodynamic approach. The main purpose of his calculation was to examine whether the existence of two-or three-phase regions existing in the ternary system could be understood simply from binary data. By making use of the regular solution approximation to evaluate the thermodynamic properties of mixing of the body centred (bcc) and face centred cubic (fcc) phases over the entire composition range, his calculations led to results which were in quite close agreement with the experimental information. He also made use of what has been designated as the "lattice stability" of chromium, which is the Gibbs energy change of chromium between the two structural states, stable bcc and metastable $\mathrm{fcc}$, the value being derived from a thermodynamic analysis of the solubility of that element in the coppernickel and copper-chromium binary systems. Lattice stability values for many of the elements, in particular for transition metals, have subsequently been evaluated by Kaufman ${ }^{2}$ ) from high pressure measurements or from the analysis of thermochemical and phase diagram data.

Meijering's pioneering work was followed a few years later by important contributions to the calculation of multicomponent phase diagrams. These contributions were due to the significant progress which had been made in the application of thermodynamic principles to the establishment of phase relationships, to improvements in describing mixing behaviour in multicomponent systems, to obtaining consistent sets of thermodynamic and phase diagram data by optimisation techniques, and to the development of computer software. Ten years ago, Hillert ${ }^{3}$ ) had already emphasized the importance of the calculation of phase equilibria in the field of phase transformations thanks to the development of computer techniques. Phase diagram calculations are being applied extensively now in various fields, e.g. semiconductors, ferrous alloys, super-alloys, ceramics salt mixtures, reactor technology, etc.

\section{Multicomponent Systems}

In order to calculate a phase equilibrium in a multicomponent system, the Gibbs energy of formation of each individual phase, stable and metastable, has to be known as a function of temperature and composition, the pressure being kept constant for most metallurgical applications. The Gibbs energy of formation of solution phases can be expressed in two terms: the Gibbs energy of formation of an "ideal" solution and the excess Gibbs energy. The first term is related to the ideal positional entropy, which is derived from a model of random mixing of an element on a given lattice site; different analytical expressions can be obtained and they depend on the structure of the phase.

For metallic solid solution, such descriptions are realistic. For metallic liquids, most of the models assume a "quasi lattice" for the structure. But for certain systems exhibiting stoichiometric phases, such as those formed by iron, cobalt and nickel with sulphur, or for systems based on elements of groups II and VI, where the chemical potentials of the constituents in the liquid phase vary drastically in the vicinity of the composition of the stoichiometric phase, two thermodynamic models have been used until now.

One of the models assumes the liquid phase to be formed by two sub-lattices, where defects are introduced in order to allow a deviation in composition from the stoichiometric value. This is equivalent to models used to describe ionic solutions. Such models in fact treat the liquid phase in the same way as a solid phase. The second model assumes that molecular species of high stability exist in equilibrium in the liquid phase.

Although the generalization of sublattices to multicomponent systems appears to be straightforward, this is not the case for the so-called "associated models", where the choice of different types of ternary compound remains hazardous. A description of the thermodynamic behaviour of molten sulphides, for example as in the case of the copper-nickel-sulphur system, has been realized by assuming the different species $\mathrm{Cu}_{2} \mathrm{~S}, \mathrm{CuS}, \mathrm{Ni}_{3} \mathrm{~S}_{2}$ and $\mathrm{NiS}$ in the liquid state.

The excess Gibbs energy represents the sum of different contributions with respect to composition. These are due to chemical and physical effects, e.g. high stability of the liquid phase, magnetic effects or chemical ordering in the solid phase. In the case of the copper-nickel-sulphur system quoted earlier, the non-ideal behaviour is described by a power series expansion with respect to composition, the values of the coefficients being fitted to the experimental values. Both models have led to very satisfactory agreement between the experimental and calculated thermodynamic properties of liquid phases. Quite recently, Nishizawa ${ }^{4}$ ) et al. have described the Gibbs energy of bcc iron-zinc (and ironchromium) solid solutions in terms of magnetic and non-magnetic components. The regular solution theory is used to evaluate the non-magnetic part of the excess Gibbs energy whereas the magnetic part is formulated according to methods described by Zener ${ }^{5}$ ) and Hillert ${ }^{6}$ ). For the iron-zinc system, a metastable miscibility gap (dashed lines) can be calculated as shown in Fig. 1. The diagram displays a sharp horn protruding along the Curie temperature line. If this horn is metastable in the binary system, addition of cobalt allows it to be stabilized and observed experimentally. Fig. 2 presents the calculated isothermal section at $1038 \mathrm{~K}$ of the cobaltzinc-iron system, in which a two-phase field of the para and ferromagnetic solid solutions is shown.

\section{Cluster Variation Method}

The cluster variation method developed by Kikuchi $^{7}$ ) has recently been used for ternary phase diagram calculations of systems exhibiting ordered phase regions which interact with miscibility gap boundaries. In this method, the distribution variable is a basic cluster of lattice points for which an approximation can be a four point squareshaped cluster, or an eight point cubeshaped cluster. For face-centred-cubic phases, the basic cluster can be a four point tetrahedron, which is better than the pair approximation. The Gibbs energy of the system is expressed in terms of these basic distribution variables corresponding to the probability of finding an i-j pair of any pair of nearest-neighbouring lattice points.

This method has recently been applied to determine isothermal sections for coherent equilibria in the copper-silver-gold system, where both ordering and clustering equilibria and their mutual interactions have been calculated from an analysis of the corresponding binary phase diagrams. It should be pointed out that this method uses only the three binary pair interaction parameters as input data and that the ther- 


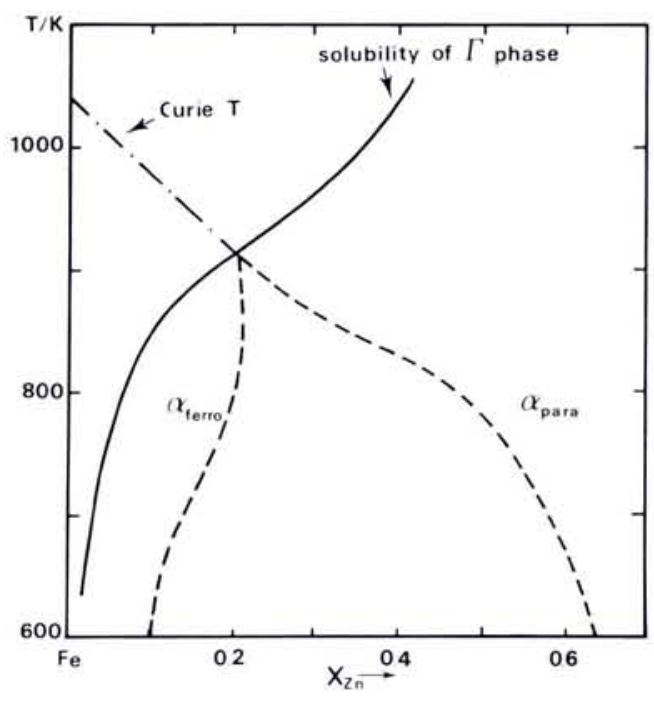

Fig. 1 - Above, Phase diagram of the iron-zinc system. The dashed lines represent the metastable phase boundaries between the $\alpha$ (ferro) and $\alpha$ (para) phases.

Fig. 2 - Right, Isothermal section of the iron-zinc cobalt system at $1038 \mathrm{~K}$

modynamic properties of the system are described by a single Gibbs energy function. The calculated sections have provided explanations for recent experimental observations of ordering and spinodal decomposition in this system. Nevertheless, from a computational point of view, this method is rather costly because of the great number of variables which have to be taken into account.

\section{Heats of Formation Models}

Physical models have been developed to evaluate the heats of formation of alloys and compounds. Van der Rest ${ }^{9}$ ) et al. have used the coherent-potential approximation to determine these thermodynamic quantities, whereas Boom et $\mathrm{al}^{10}$ ) developed a semi-empirical equation. The heats of formation according to the latter model are related to the electro-negativity difference of the elements and the charge-density mismatch at the Wigner-Seitz boundary. Two parameters are required and these are derived from experimental information. Quite recently, Pettifor ${ }^{11}$ ) has presented a microscopic theory of transition-metal alloys based on the tight-binding approximation, for which no adjustable parameters are needed. His theory gives values in good agreement with those obtained from Boom et al's equation.

Models derived from statistical thermodynamics, such as the regular solution model ${ }^{12}$ ), the quasi-chemical model ${ }^{13}$ ) or the surrounded atom model ${ }^{14,15}$ ), have been used extensively to describe the heats and excess Gibbs energies of multicomponent metallic liquid solutions. They lead to values which are generally in good agreement with the experimental data. In these models the parameters are derived from binary data.

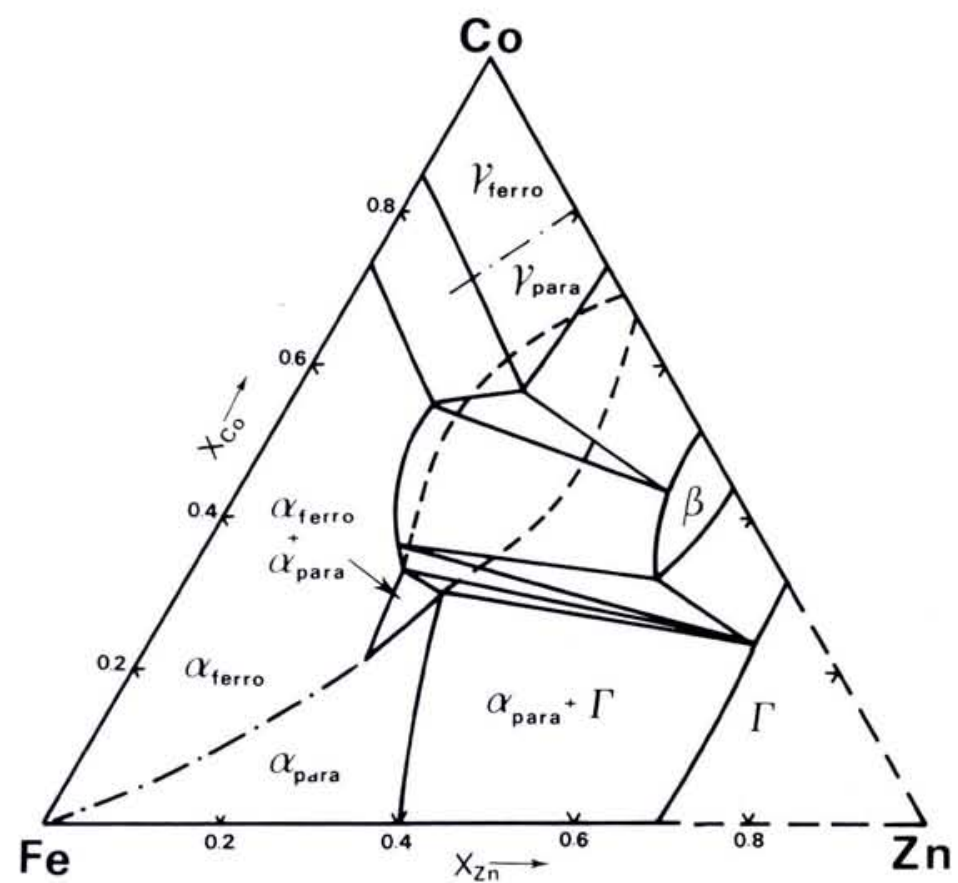

For many multicomponent systems in which the interactions between the constituents are not strong, the excess properties of the ternary solution phases can also be adequately described by empirical equations such as power series, or by geometrically weighted values of the excess Gibbs energy of mixing of the limiting binary systems, for which more experimental information is available. The most widely used equations for phase diagram calculation have been described in a review article ${ }^{16}$ ).

\section{Diagram Determination}

Phase diagrams are generally determined by classical methods such as thermal analysis, X-ray investigations, equilibrium techniques etc, for relatively small composition and temperature ranges. Thermodynamic quantities are generated by completely different experimental techniques. Although these two classes of data acquisition have developed separately through the work of different scientists, the derived data can nevertheless be reconciled through thermodynamic principles to ensure a complete and consistent description of the system. This is why optimization techniques are used more and more to obtain consistent sets of thermodynamic and phase diagram data. Experimental information for partial Gibbs energies, heats of formation, specific heats, solubility measurements etc. are the input data required to produce a set of equations expressing the composition dependence of these quantities as well as the equilibrium relationships between the phases, the thermodynamic parameters being derived by a least square method.

These techniques allow the evaluation of the thermodynamic properties of metastable or hypothetical solutions or compounds, for example the different iron carbides of the types $\mathrm{Fe}_{23} \mathrm{C}_{6}$ or $\mathrm{Fe}_{7} \mathrm{C}_{3}$. Hence a thermodynamic description can be derived

\section{GRAZ}

FULL PROFESSOR OF THEORETICAL PHYSICS

The TECHNISCHE UNIVERSITÄT GRAZ (AUSTRIA) invites applications for the position of a full professor for its Institute of Theoretical Physics. The position is to be filled on or about 1 October 1981. The candidate's research field is expected to be in one or more of the following areas: Atomic-Nuclear Energetics and Conversion, Electromagnetic Theory, Mathematical Physics, Theoretical Solid State Physics. He should be an experienced teacher of theoretical physics and able to interact with researchers in the above named areas as well as in Radiation Physics.

Applicants are requested to submit a bibliographical sketch, list of publications, and summary of their teaching and research experiences to the :

DEKAN DER TECHNISCH-NATURWISSENSCHAFTLICHEN FAKULTÄT DER TECHNISCHEN UNIVERSITÄT GRAZ, Petergasse 16, A-8010 GRAZ, AUSTRIA, before 31 January 1981. 
of stoichiometric phases of the type $(\mathrm{Cr}$, $\mathrm{Fe})_{23} \mathrm{C}_{6}$ or $(\mathrm{Cr}, \mathrm{Fe})_{7} \mathrm{C}_{3}$, where iron substitutes chromium in these stable carbides.

A knowledge of the thermodynamic properties of the elements, stoichiometric compounds and solution phases, in stable or metastable states then allows the determination of phase relationships either for stable or metastable equilibria. The compositions of the phases in equilibrium at a given temperature and pressure are obtained by minimizing the Gibbs energy with respect to the independent variables, or by resolving a set of equations expressing the equality of the chemical potentials of the different constituents in these phases.

\section{Motivation}

The main interest in phase diagram calculations using a thermodynamic approach is that from a limited amount of information concerning the mixing properties of solution phases, equilibrium phase relationships and compositions can be established from a limited number of experiments. Successful results have been obtained in applying such calculations to crystal growth of semiconductors by liquid phase epitaxy, or to directionally solidified in situ super-alloy composites. Different representations can be used; isothermal sections of ternary systems, or vertical cuts corresponding to a quasi-binary section defining temperature versus metallic ratio, are the most classical ways of representation of equilibrium diagrams. A simple geometrical transformation can be made to plot the phase limits in a chemical potential (logarithm of the partial pressure or the activity of any of the constituents) versus atom ratio diagram. These types of diagram have the advantage of allowing phase boundaries of an isothermal section of a ternary system to be plotted in a rectangular diagram, similar to that used to represent binary systems.

Problems arise of course when the number of constituents is greater than three, but numerical techniques for calculating phase boundaries in higher order systems are now being developed in many laboratories together with graphical methods for representing the results.

\section{Basic Data Sources}

To provide the best possible phase diagram it is essential, as mentioned above, to obtain consistent sets of thermochemical and phase diagram data. Members of the international Calphad project produce on a regular basis, assessed thermodynamic data and phase diagrams for selected binary and ternary systems.

Computer based databanks providing thermochemical data for inorganic substances are also in operation in Europe. The scientific group Thermodata Europe, which is formed by members of AERE, Harwell
(UK) NPL, Teddington (UK), RheinischWestfälische Technische Hochschule, Aachen (FRG), Royal Institute of Technology, Stockholm (S), IRSID, Maizières les Metz (F), L.T.P.C.M. - Institut National Polytechnique and Thermodata (in France) at Saint-Martin-d'Hères (F) offers critically assessed and up-dated data and in certain cases also, an on-line service.

A more ambitious goal is assigned to Codata Task Group on the Systematization and Internationalization of Thermodynamic Data. This Task Group is charged with the development of a world-wide system for producing self-consistent tables, whether they originate from data centres or from groups with special interests in special areas. The task involves systematic analysis of the methodology of obtaining such tables for special classes of substances (alloys, aqueous solutions...).

These international efforts reflect the need to obtain compiled and assessed data for given substances and to develop interactive procedures to facilitate data bank operations. At the same time, great attention should be paid to educating the technical and scientific community to the efficacy of this development.

\section{BIBLIOGRAPHY}

1. Meijering J.L., Acta Met. 5 (1957) 275.

2. Kaufman L. and Bernstein H., Computer Calculations of Phase Diagrams (Academic Press, N.Y.) 1970.

3. Hillert M., Phase Transformations (A.S.M. Metals Park, Ohio) 1970, p. 181.

4. Nishizawa T., Hasebe M. and Ko M., Acta Met. 27 (1979) 817 and Proc. CALPHAD VIII (Stockholm) 1979.

5. Zener C., Trans. AIME 203 (1955) 619.

6. Hillert M., Wada T. and Wada H., J. Iron Steel Inst. 205 (1967) 539.

7. Kikuchi R., Acta Met. 25 (1977) 195.

8. Kikuchi R., de Fontaine D., Murakami M. and Nakamura T., Acta Met. 25 (1977) 207.

9. Van der Rest J., Gautier F. and Brouers F., J. Phys. F. Metal Phys. 5 (1975) 2283.

10. Boom R., de Boer F.R. and Miedema A.R., J. Less Common Metals 46 (1976) 46.

11. Pettifor D.G., Phys. Rev. Lett. 42 (1979) 13 , 846.

12. Hildebrand J.H., J. Am. Chem. Soc. 51 (1929) 51.

13. Guggenheim E.A., Mixtures (Clarendon Press, Oxford) 1952.

14. Mathieu J.C., Durand F. and Bonnier E., J. Chim. Phys. 62 (1965) (11-12) a) 1289, b) 1297.

15. Lupis C.H.P. and Elliott J.F., Acta Met 15 (1967) 265

16. Ansara I., Intern. Metals Review 1 (1979) 20.

\section{Atomic Spring in Heidelberg}

The First European Conference on Atomic Physics (ECAP) of the European Physical Society will take place in Heidelberg, FRG, from 6 to 10 April, 1981. All Sections of the Atomic Physics Division will be represented: Atomic Spectroscopy, Molecular Physics, Electron and Atomic Collisions as well as Chemical Physics. The Conference has been initiated with the aim of bringing more closely together the various branches of atomic and molecular physics, existing in Europe, underlining the common scientific aspects and enhancing the cooperation between European laboratories. In order to support this idea the national physical societies have dispensed with their own scientific meetings in 1981 in favour of ECAP.

The Atomic Physics Division is responsible for the scientific organization of the meeting; G. zu Putlitz (University of Heidelberg and Gesellschaft für Schwerionenforschung, Darmstadt) is Conference Chairman. The scientific programme calls for five morning sessions and four afternoon sessions. In each morning session two plenary lectures will be given followed by two parallel sessions with two progress reports each. The plenary lectures will be of general interest for all participants. They should, as well as the progress reports, deal with subjects in the fields mentioned above so that young physicists and students get acquainted with the problems.
The programme discussion concentrates on the following focal points: weak interactions between atoms and molecules, laser chemistry, reactive scattering and chemical ionization, atomic and molecular collision processes in combination with selective excitation of states, coincidence experiments, scattering processes in high fields, photochemistry in the upper atmosphere, high resolution atomic and molecular spectroscopy in particular with respect to fundamental questions of atomic physics, the use of accelerators and synchrotron radiation in atomic and molecular physics, atomic physics in heavy ions. The afternoon sessions will be devoted to contributed papers with sessions for oral as well as poster presentation.

Conference abstracts are limited to two pages and have to be typed on forms in order to be directly reproducible. The forms are obtainable from the secretary's office. Deadline is 1 February, 1981. The conference address is:

European Conference on Atomic Physics (ECAP), Professor Dr. G. zu Putlitz, Physikalisches Institut der Universität Heidelberg, Philosophenweg 12, D-6900 Heidelberg. Telephone (6221) 569-211, -212, -213; Telex 461515 unihd d.

Two Conference Circulars are mailed already and can be ordered from the abovementioned address.

\section{R. Neumann, Heidelberg}

